\title{
The Possible Impacts of Climate Change on Fermilab's Radioactive Air Emissions
}

Anne Murray, Purdue University; Matthew Quinn, Environmental Health and Safety Section (ESH), Fermilab;

Diane Reitzner, ESH, Radiation Physics Science, Fermilab

\section{Background}

Climate change was a natural process that is now accelerated and amplified by human activity through the Greenhouse Effect. This effect describes how, when sunlight reaching Earth's atmosphere passes to its surface, it is absorbed and then radiated upwards in the form of infrared heat. About $90 \%$ of this heat is then absorbed by greenhouse gases (GHG) and radiated toward the surface, increasing temperatures. Human industrial activities, such as the combustion of fossil fuels, have increased the concentrations of GHG in Earth's atmosphere, amplifying the Greenhouse Effect and climate change.

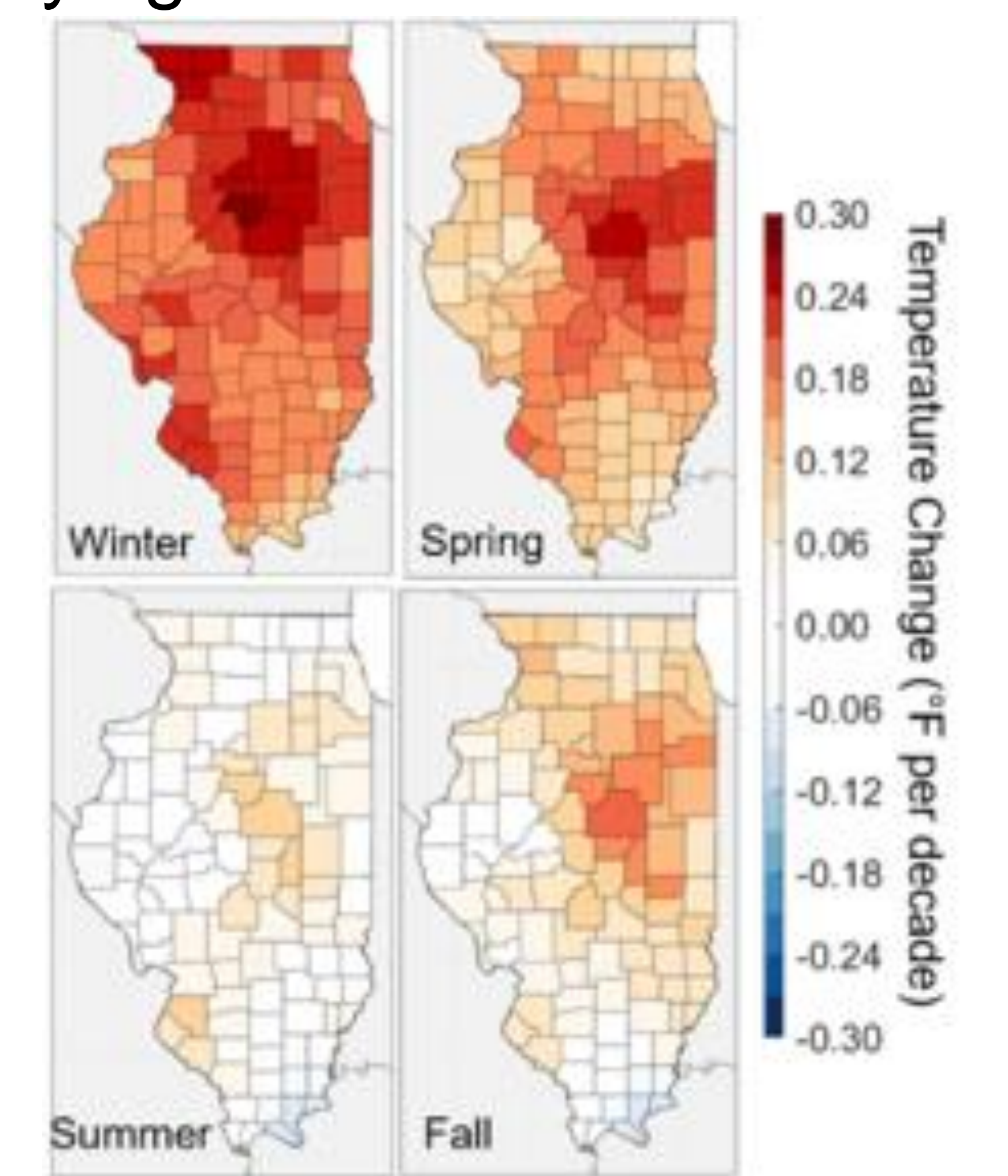

These maps show trends in the daily average temperatures per decade, season, and county in Illinois. These trends were calculated using observations from $1900-2020$
show that increased temperatures are concentrated in Northern Illinois in winter. Climate change alters atmospheric conditions, affecting the distribution of airborne particles, including the radioactive isotopes Fermilab emits while running accelerators. Different wind patterns may increase the population's exposure to these isotopes, impacting Fermilab operations Since Fermilab hopes to increase accelerator power in the future while ensuring public safety, understanding the impact of climate change on the distribution of these emissions is vital for Fermilab's future.

\section{Acknowledgements}

This manuscript has been authored by Fermi Research Alliance, LLC under Contrac No. DE-AC02-07CH11359 with the U.S. Department of Energy, Office of Science, Office of High Energy Physics.

\section{References}

EPA. (2021, February 25). CAP-88. EPA. https://www.epa.gov/radiation/cap-88-pc-version-41 downloads-and-supporting-documents

Zobel, Z., Wang, J., Wuebbles, D. J., \& Kotamarthi, V. R. (2017, December 18). High-Resolution dynamical DOWNSCALING ENSEMBLE projections of future extreme Temperature distributions fo the United States. AGU Journals

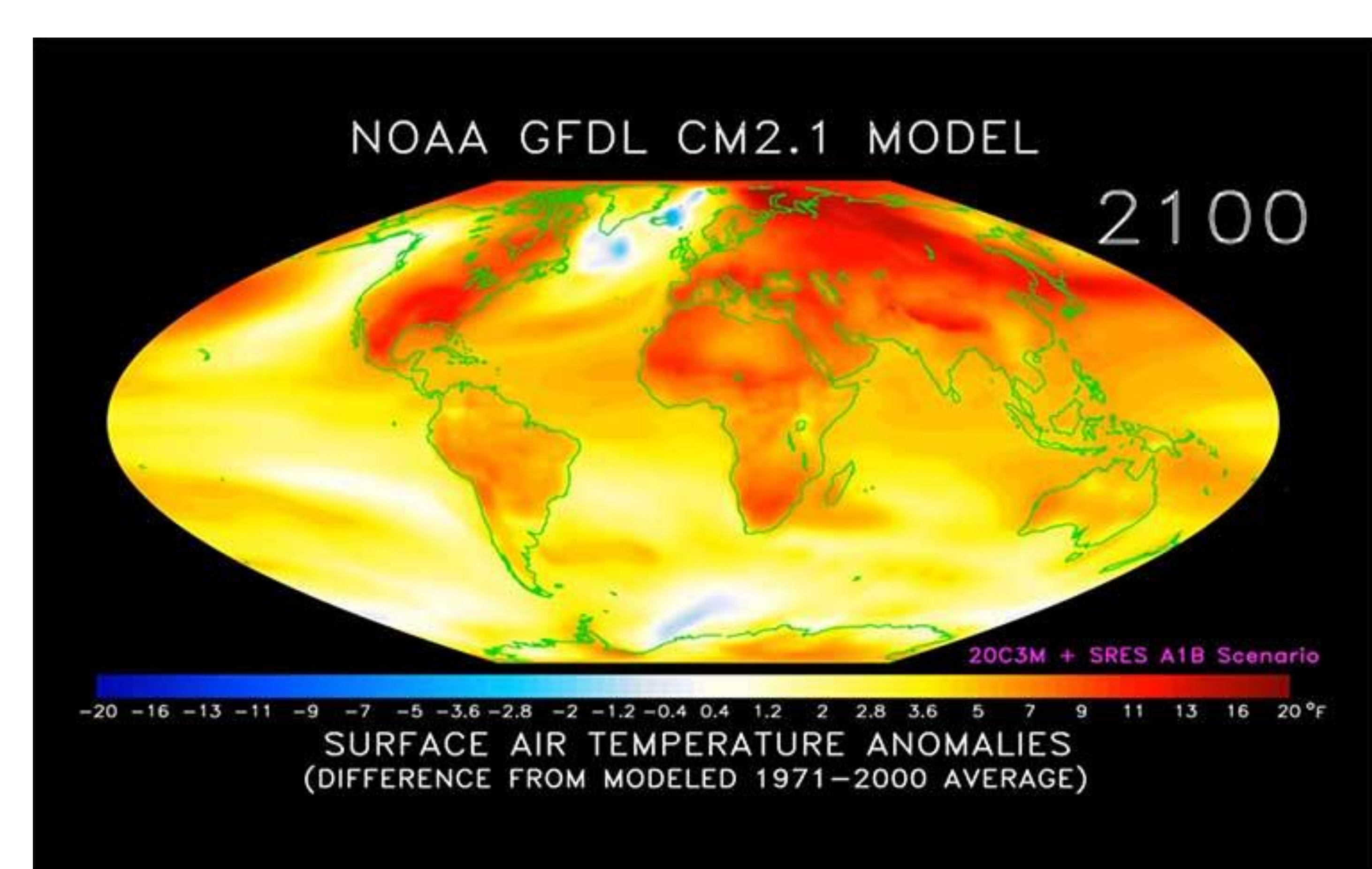

Temperature projections compiled from the NOAA climate model

Climate Modeling

Climate models are computer simulations that summarize Earth's climate systems. They calculate atmospheric conditions, temperature, pressure, wind patterns, and humidity, enabling projections of long-term climate patterns and weather events. The climate model used in this study was developed by the University of Illinois and predicts these variables in the Fermilab region for the middle and end of this century (Zobel, 2017).

\section{CAP-88}

CAP-88 is a US EPA computer model used as a compliance tool under the National Emissions Standard for Hazardous Air Pollutants (EPA, 2021). Its purpose is to estimate the public's dosage and risk from airborne radionuclide emissions. It uses momentum-based Gaussian Plume Modeling to estimate the dispersions of these particles and evaluates the low-level chronic exposure the public may experience under varied climactic and weather conditions.

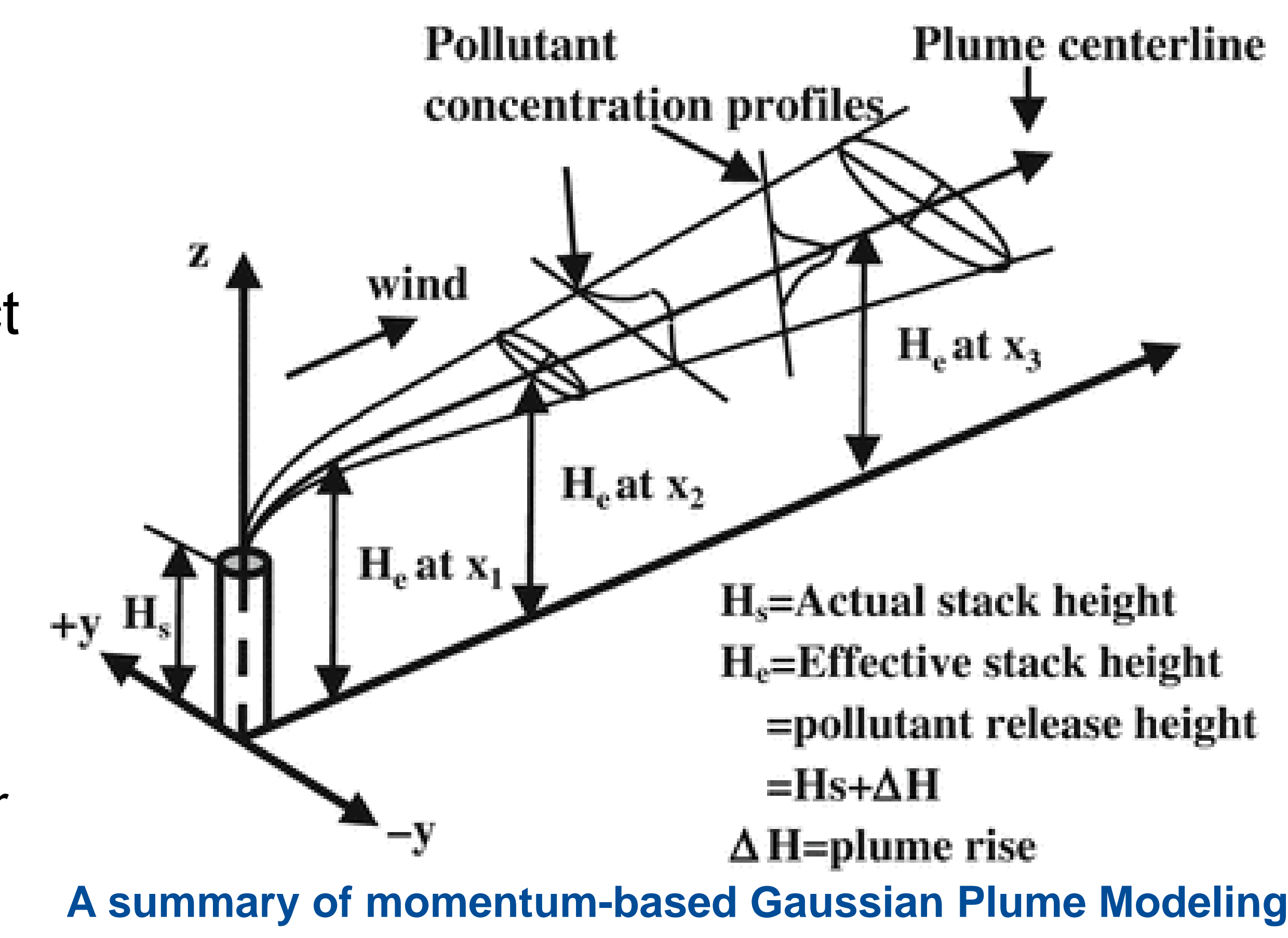

Results

Four scenarios were devised: mid-century and end-century decades with a $4.5^{\circ} \mathrm{C}$ temporal increase, and mid-century and end-century decades with an $8.5^{\circ} \mathrm{C}$ temporal increase. The maximumly-exposed individual shares the same location across all 4 scenarios at 800 meters northwest from campus. As distance from Fermilab increases, the dosage decreases, as shown by the collective dosage figure. Every scenario shared the same conclusion: Fermilab's radioactive air emissions will remain far under the EPA threshold, never surpassing $10 \%$ of the federal limit. This means Fermilab operations will not be impacted by extreme climate changes in the long-term and that the lab has ample opportunities to increase power to support future projects safely.

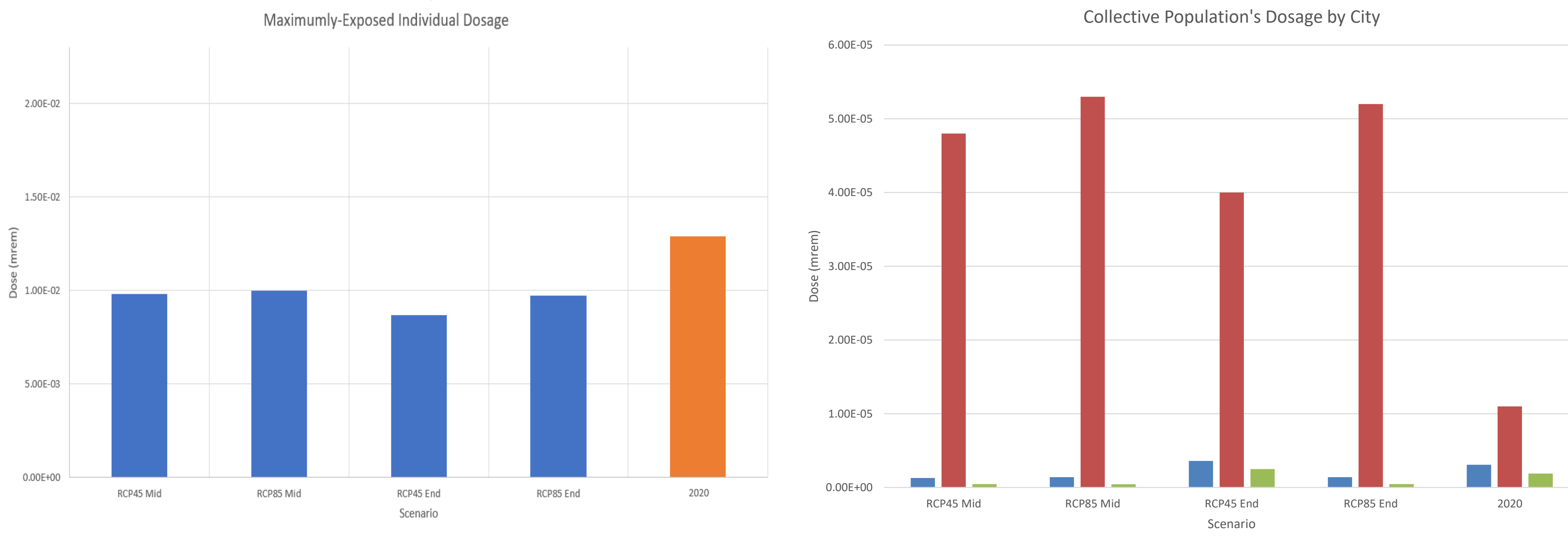

The maximumly-exposed individual's (MEI) total radionuclide exposure for all 4 scenarios
a

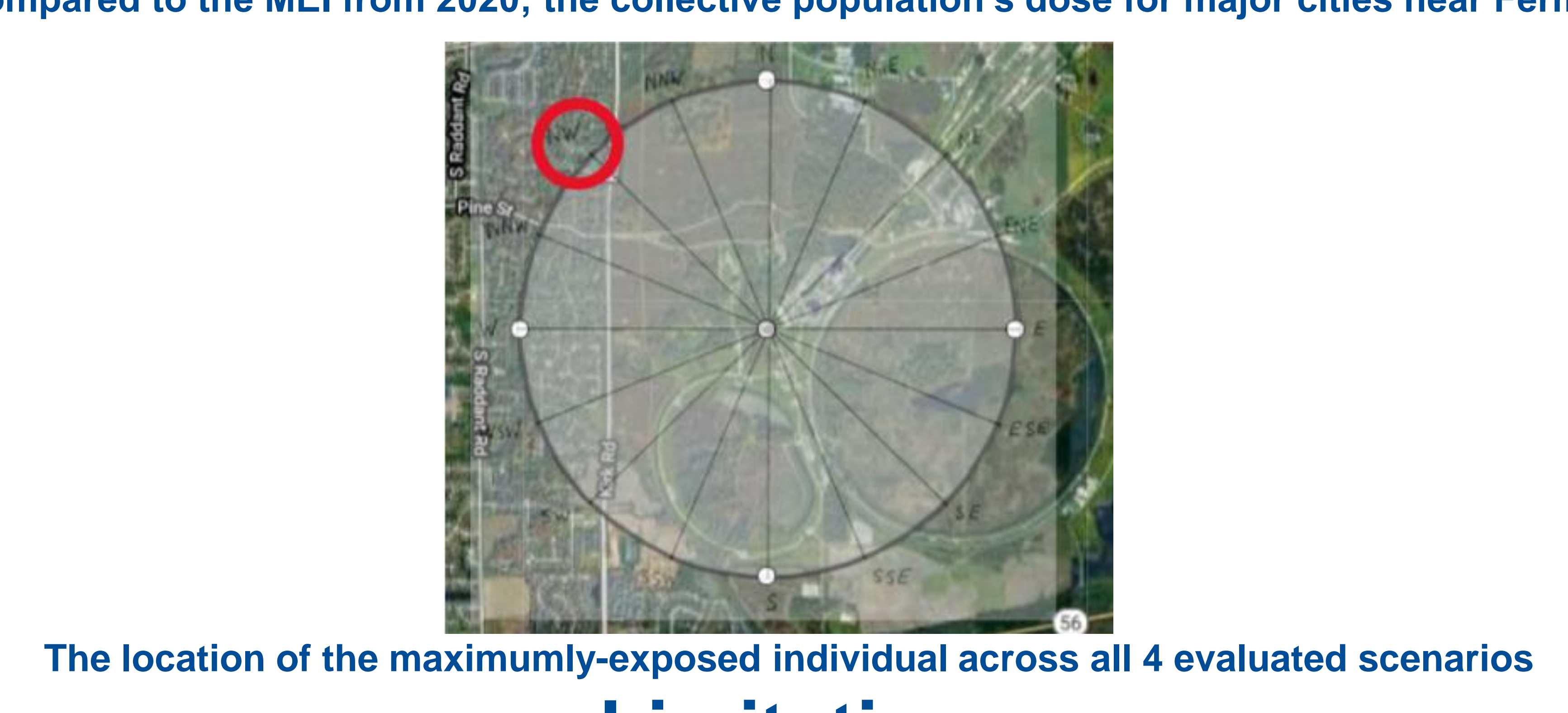

Limitations

Predicting climate and weather patterns nearly 80 years from now has substantial room for error. The most pressing limitation will be how human actions change GHG levels over the century, as the temporal increase will be proportional to these emissions. These emissions may increase with a growing population, or they may decrease with improved efficiency and regulation. A second limitation lies within the programs used for these calculations, as they required simplifying the climate model's data through averages and were only capable of devising two temperature scenarios. 